\title{
Incidencia de los ritmos musicales en la movilidad articular de los adultos mayores de la ciudadela hospitalaria, ubicada en el cantón Rumiñahui
}

DOI: https://doi.org/10.33262/ap.v2i3.32

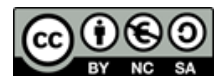

Incidence of musical rhythms in the joint mobility of older adults of the hospital ciudadela, located in the canton Rumiñahui

\section{Vanessa Lucia Ochoa Sangurima., ${ }^{1}$ Mónica Cabezas Cabezas Flores., ${ }^{2}$ Orlando Rodrigo} Carrasco Coca., ${ }^{3}$ \& Evelyn Siomara Ronquillo Caceres. ${ }^{4}$

\begin{abstract}
.
The impact of musical rhythms was investigated in joint mobility of the elderly of the Hospitaller citadel, located in canton Rumiñahui, in the period April to August 2016, as a therapeutic means in support of basic movements as most of these people today are subjected to physical inactivity, so this research is established as an aid to movement knowing that today much importance is not given to the elderly, for which the problem arose then for the elder perform physical activity ( joint movement ) with the help of musical rhythms , in order to understand their loss or gain of articular movement angles by musical rhythms.

Keywords: Musical rhythms, joint mobility, older adults.

\footnotetext{
${ }^{1}$ Universidad de las Fuerzas Armadas - "ESPE”, Carrera de Pedagogía de la Actividad Física y Deportes. Quito, Ecuador.vane4lali@gmail.com

${ }^{2}$ Universidad de las Fuerzas Armadas - "ESPE”, Carrera de Pedagogía de la Actividad Física y Deportes. Quito, Ecuador.mmcabezas@espe.edu.ec

${ }^{3}$ Universidad de las Fuerzas Armadas - "ESPE”, Carrera de Pedagogía de la Actividad Física y Deportes. Quito, Ecuador. orcarrasco@espe.edu.ec

${ }^{4}$ Universidad de las Fuerzas Armadas - "ESPE”, Carrera de Pedagogía de la Actividad Física y Deportes. Quito, Ecuador. esronquillo@espe.edu.ec
} 


\section{Resumen.}

Se investigó la Incidencia de los ritmos musicales en la movilidad articular de los adultos mayores de la Ciudadela Hospitalaria, ubicada en el Cantón Rumiñahui, en el periodo abril - Agosto del 2016, como un medio terapéutico en la ayuda de movimientos básicos ya que la mayoría de estas personas en la actualidad se sujetan al sedentarismo, por eso esta investigación está establecida como un medio de ayuda al movimiento sabiendo que en la actualidad no se da mucha importancia al adulto mayor, para ello se planteó el problema a continuación, para que el anciano realice actividad física (movimientos articulares) con la ayuda de los ritmos musicales, con la finalidad de entender su pérdida o ganancia de ángulos de movimiento articular mediante los ritmos musicales.

Palabras claves: Ritmos musicales, movilidad articular, adultos mayores.

\section{Introducción.}

La música en su historia según Clementi da a conocer el surgimiento de la música, como fue apareciendo tomando en cuenta a la aparición de la lengua como una principal causa para desarrollo de la música sin dejar de lado que los instrumentos son los principales objetivos para que la música funcione adecuadamente como una combinación fundamental y rica para el oído. (Clementi, p.3)

La música desempeña un papel muy importante porque las personas que la crean nacen con ese don de concebir melodías adecuadas a los oídos de las personas siempre y cuando tenga conocimiento y manejo de las notas musicales, si se la pretende hacer en parejas la música cambiaria totalmente, no es lo mismo interpretarla como Rudolf Serki o Beethoven. (Guevara, p5)

Cada uno de los ritmos fue apareciendo en cada uno de los países por ello los ritmos musicales tienen origen y su vinculación con la sociedad y las necesidades que cada región la necesitaba, de esta manera cada uno de los países tienen su identidad concreta que hacen sobresalir a su gente y llenarse de orgullo para ello crearon y comparten al mundo entero sus riquezas, sus formas de vida. (Morales, p.3)

Los ritmos musicales son aquellos que provienen de un arreglo sonoro que deleita la sensibilidad auditiva, crea vida a la música acotando su percepción de cualidades que difieren en cada ritmo musical. Centramos nuestra atención sobre estímulos que se repiten, idénticos a ellos mismos, a intervalos iguales de tiempo. Estas estimulaciones se organizan para nosotros en grupos. (Schloezer, p.2)

Se define que la movilidad es entendida como la necesidad de moverse, por tanto, un derecho social que es necesario preservar y garantizar en forma igualitaria, es también 
movilizarse de un lugar a otro en determinados tiempos, conjuntamente con la ayuda del cuerpo en general de sus articulaciones, huesos. (Valladolid, p7)

La articulación es aquella que proporciona elasticidad y plasticidad al cuerpo, es también la unión entre dos partes que integran una máquina, que posibilita y ordena su movimiento interno se lo denomina como articulación, el cuerpo humano tiene articulación que proporcionan el movimiento. (Liekens, p.1)

El envejecimiento es un proceso que no sólo afecta a las personas, sino que también ocurre en las poblaciones, y es lo que se llama el envejecimiento demográfico.es aquella persona que tiene decadencia de su movilidad con el pasar de los años y va careciendo de conocimientos y pérdida de memoria. (Catarina, p4, 5)

Por lo general las personas de la tercera edad a las que erróneamente la sociedad las margina tienen en conjunto características que los limita como seres humanos sin considerar que son un bagaje de experiencias por ello tenemos, enfermedades biológicas (artrosis, diabetes, cataratas, párkinson osteoporosis, y cardiovasculares). Enfermedades neurológicas o mentales (el alzhéimer, demencia senil). (Hernández, p.1)

\section{Material y Métodos.}

\section{Investigación Correlacional}

Este tipo de investigación está indicada para determinar el grado de relación y semejanza que pueda existir entre dos o más variables, es decir, entre características o conceptos de un fenómeno. Ella no pretende establecer una explicación completa de la causa - efecto de lo ocurrido, solo aporta indicios sobre las posibles causas de un acontecimiento. (Gonzales, 2005, p5)

Relacionar, y a la vez verificar la semejanza de las variables tanto dependientes como independientes, los cuales permiten darse cuenta si es una causa o un efecto con las características de los diferentes acontecimientos.

\section{Métodos de investigación.}

\section{Método hipotético-deductivo.}

El método hipotético-deductivo es el procedimiento o camino que sigue el investigador para hacer de su actividad una práctica científica, lo cual obliga al científico a combinar la reflexión racional o momento racional la formación de hipótesis y la deducción, con la observación de la realidad o momento empírico (la observación y la verificación). 
(Sánchez, 2009,p3)

El autor nos permite ver los diferentes caminos o los procedimientos que un investigador tiene que hacer para alcanzar una práctica científica, con elementos fundamentales como son la hipótesis y la deducción dando así herramientas que permitan la observación y la verificación de lo investigado.

\section{Método-sintético}

Es un proceso mediante el cual se relaciona hechos aparentemente aislados y se formula una teoría que unifica los diversos elementos. Consiste en la reunión racional de varios elementos dispersos en una nueva totalidad, este se presenta más en el planteamiento de la hipótesis. El investigador sintetiza las superaciones en la imaginación para establecer una explicación tentativa que someterá a prueba. (Orozco, 2003, p8)

El método sintético nos permite relacionar varios elementos dispersos siendo estos una parte fundamental para llegar al todo, es decir que los datos sintéticos es un subconjunto de datos anónimos, donde utiliza en una variedad de campos como un filtro para la información.

\section{Método-Analítico-Sintético.}

Se distinguen los elementos de un fenómeno y se procede a revisar ordenadamente cada uno de ellos por separado. La física, la química y la biología utilizan este método; a partir de la experimentación y el análisis de gran número de casos se establecen leyes universales. Consiste en la extracción de las partes de un todo, con el objeto de estudiarlas y examinarlas por separado. (Fernández, 2006, p10)

Este método nos permite llevar un orden lógico, es decir revisar ordenadamente cada uno y por separado descomponiéndolo en sus partes o elementos para observar las causas, la naturaleza y los efectos dando así lugar al análisis, la observación y examen de un hecho en particular.

\section{Instrumentos de medición.}

\section{Encuesta.}

Se busca recaudar datos por medio de la encuesta dirigida al adulto mayor con respecto a cómo influyen los ritmos musicales en la movilidad articular, aplicando preguntas cerradas dirigida a la población estadística, con el fin de conocer estados de opinión, características o hechos específicos. Seleccionar las preguntas más convenientes, de acuerdo con la naturaleza de la investigación. (Johnson \& Kuby, parr.1)

Test para evaluar la actividad funcional de los adultos mayores 
Este test que vamos aplicar tiene el nombre de Senior Fitness Test, que es la agrupación de varios tipos de test que miden la flexibilidad-movilidad articular cada uno elegido con mucha precaución ya que va dirigido a las personas de la tercera edad con las cuales hay que tener mucha precaución. (Ramos, p9)

Goniometría (medir ángulos) El instrumento que se usa con mayor frecuencia es el goniómetro de dos ramas, para medir la amplitud articular se sitúa en unos puntos de referencia óseos o siguiendo los ejes longitudinales corporales. Se basan en el principio de la indicación permanente de la vertical, por ello este tipo de goniómetro siempre debe colocarse vertical, no es necesario hacer coincidir el centro del goniómetro con el centro articular. (Sadhanad, p12)

\section{Población.}

La investigación va dirigida a los adultos mayores del Barrio, Ciudadela Hospitalaria, la muestra de la población es de 20 personas que realizan actividad física los sábados a las siete de la mañana: $\mathrm{P}=20$

\section{Recolección de Datos o Información.}

Solicitamos la autorización al Señor que está a cargo del grupo al que vamos a realizar los estudios, su respuesta fue positiva por lo que vamos a realizar la aplicación de la encuesta y la ficha de observación, para con ello poder tener un panorama de la situación en la que se encuentran los adultos mayores.

La recolección de datos se la va realizar mediante una encuesta, una ficha de observaciones que se les aplicara a los adultos mayores de la Ciudadela Hospitalaria, ubicada en el Cantón Rumiñahui, en el periodo abril - Agosto del 2016.

\section{Tabulación de Información.}

Luego de realizar las encuestas y las fichas de observación a los adultos de la Ciudadela Hospitalaria, ubicada en el Cantón Rumiñahui, procederemos a tabular los resultados utilizando el programa Excel y la representación gráfica de pasteles que arrojarán los porcentajes para tener un dato verídico de la situación en la que ellos se encuentran.

Determinar el porcentaje de incidencia de los ritmos musicales en la movilidad articular del adulto mayor de la Ciudadela Hospitalaria, ubicada en el Cantón Rumiñahui, en el periodo abril - Agosto del 2016, con la finalidad de entender su pérdida o ganancia de ángulos de movimiento articular mediante los ritmos musicales. 


\section{Análisis de resultados.}

Tabla 1: Senior Fitness Test

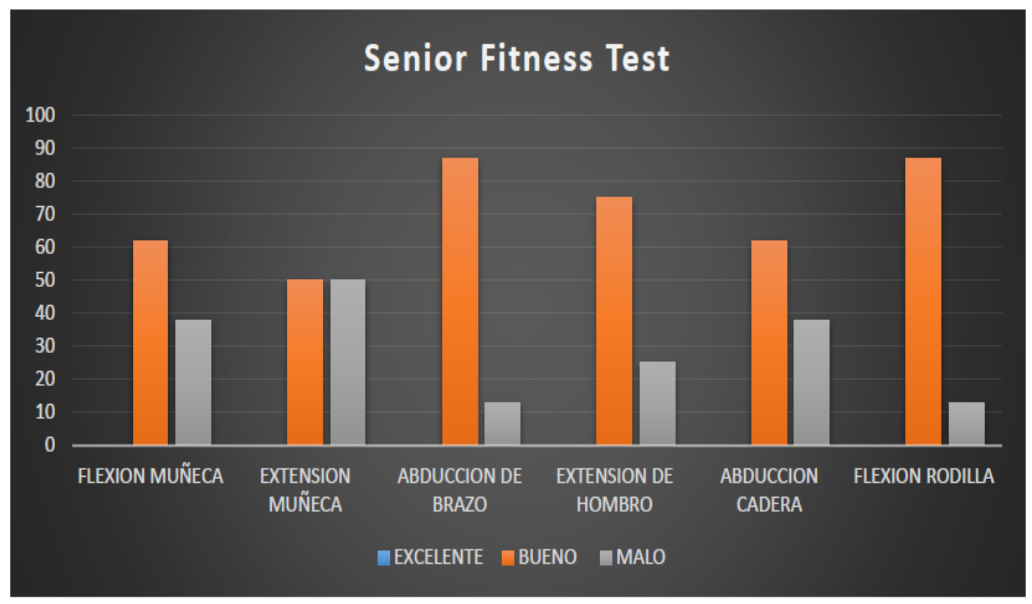

Fuente: Elaboración propia.

Tabla 2: Test Goniométrico

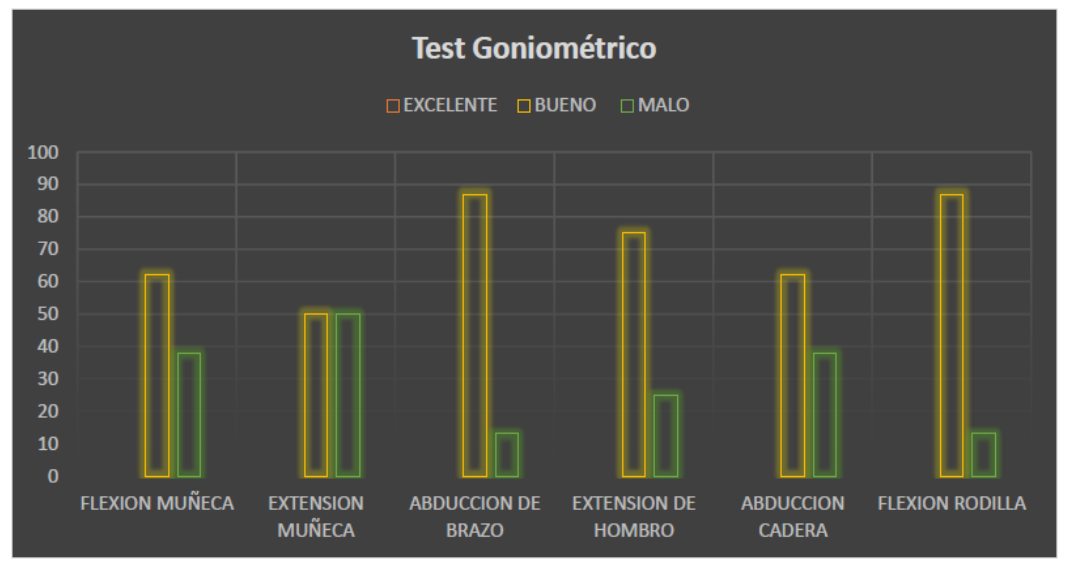

Fuente: Elaboración propia.

\section{Conclusiones.}

- De acuerdo a los análisis aplicados del test Senior Fitnes y test goniométrico, podemos observar ciertas mediciones tanto en mujeres como hombres de la tercera edad (60-76años), los resultados arrojados son aceptables en la movilidad articular en algunos adultos mayores, al contrario de otros, cabe recalcar la menor cantidad de ellos los cuales muestran dificultades en su movilidad articular empleados en los respectivos test, se deduce que es por los problemas propios de su edad, por lo cual se podrá realizar el proyecto aplicando ejercicios aptos para ellos y mantener el grado de movimiento. 


\section{Referencias bibliográficas.}

Asisten, Juan Carlos, F. D. (1999). http://coleccion.educ.ar. Obtenido de http://coleccion.educ.ar/coleccion/CD13/contenidos/materiales/archivos/sonido.pdf

Beltrán. (1972). www.balletfolkecuador.com. Obtenido de www.balletfolkecuador.com: http://www.balletfolkecuador.com/index_uno.html

Carrión, Doris Solís F. S. (2013). http://www.inclusion.gob.ec. Obtenido de http://www.inclusion.gob.ec/wpcontent/uploads/downloads/2012/09/Agendas_ADULTOS.pdf

García. (1950). www.wikipedia.org. Obtenido de www.wikipedia.org: http://es.wikipedia.org/wiki/M\%C3\%BAsica_de_Am\%C3\%A9rica_Latina

Gutiérrez. (1950). www.wikipedia.org. Obtenido de www.wikipedia.org: http://es.wikipedia.org/wiki/Ritmo

González, C. M. (2010). http://www.fenercom.com. Obtenido de http://www.fenercom.com/pdf/publicaciones/Movilidad-Urbana-Sostenible-un-retoenergetico-y-ambiental-2010.pdf

Sanín, J. S. (2010). http://www.teoria.com/. Obtenido de http://www.teoria.com/articulos/guevara-sanin/guevara_saninteoria_de_la_musica.pdf

Sans, J. F. (1948). Definiciones del Ritmo Musical. Obtenido de http://www.academia.edu/2556635/Definiciones_del_ritmo_musical_Andre_Souris

Sans, J. F. (1948). http://www.academia.edu. Obtenido de http://www.academia.edu/2556635/Definiciones_del_ritmo_musical_Andre_Souris

Serra, M. (2010). http://gemarivas.files.wordpress.com. Obtenido de http://gemarivas.files.wordpress.com/2010/01/t10-def-ritmo2.pdf

Sexto. (1953). www.juntadeandalucia.es. Obtenido de www.juntadeandalucia.es: http://www.juntadeandalucia.es/averroes/recursos_informaticos/andared01/paisaje_ so noro/sonido.html

Tobar. (1980). Wikipedia. Obtenido de La Enciclopedia Libre Wikipedia: http://es.wikipedia.org/wiki/M\%C3\%BAsica_del_Ecuador. 
Para citar el artículo indexado

Ochoa Sangurima, V. L., Cabezas Flores, M. C., Carrasco Coca, O. R., \& Ronquillo Caceres, E. S. (2020). Incidencia de los ritmos musicales en la movilidad articular de los adultos mayores de la ciudadela hospitalaria, ubicada en el cantón Rumiñahui. AlfaPublicaciones, 2(3), 13-20. https://doi.org/10.33262/ap.v2i3.32

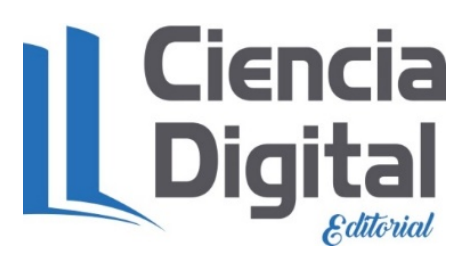

El artículo que se publica es de exclusiva responsabilidad de los autores y no necesariamente reflejan el pensamiento de la Revista Alpha Publicaciones.

El artículo queda en propiedad de la revista y, por tanto, su publicación parcial y/o total en otro medio tiene que ser autorizado por el director de la Revista Alpha Publicaciones.
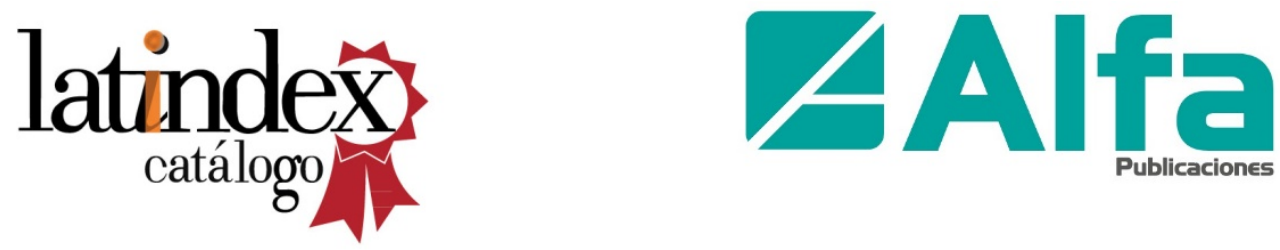